\title{
TOTAL POSITIVITY, ABSORPTION PROBABILITIES AND APPLICATIONS
}

BY SAMUEL KARLIN

Communicated by I. J. Schoenberg, August 24, 1960

A function $K(x, y)$ of two real variables ranging over linearly ordered one-dimensional sets $X$ and $Y$ respectively is said to be totally positive of order $r\left(T P_{r}\right)$ if for all $1 \leqq m \leqq r, x_{1}<x_{2}<\cdots<x_{m}$, $y_{1}<y_{2}<\cdots<y_{m}\left(x_{i} \in X ; y_{j} \in Y\right)$, we have the inequalities

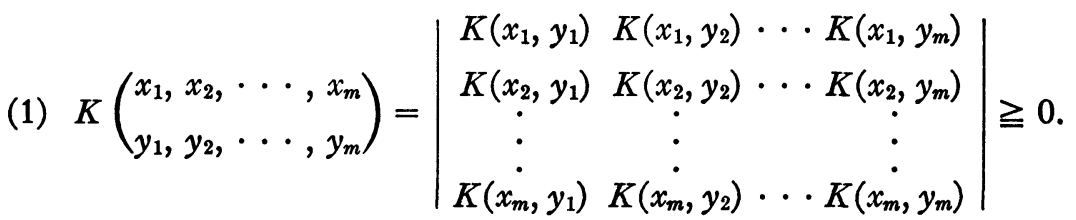

Typically, $X$ is an interval of the real line or a countable set of discrete values on the real line; similar for $Y$.

A related concept is that of sign regularity. A function $K(x, y)$ is sign regular of order $r$, if for every $x_{1}<x_{2}<\cdots<x_{m}, y_{1}<y_{2}<\cdots$ $<y_{m}\left(x_{i} \in X ; y_{j} \in Y\right)$ and $m, 1 \leqq m \leqq r$,

$$
\operatorname{sign} K\left(\begin{array}{l}
x_{1}, x_{2}, \cdots, x_{m} \\
y_{1}, y_{2}, \cdots, y_{m}
\end{array}\right)=\epsilon_{m}
$$

depends on $m$ alone.

An important specialization occurs if a $T P_{r}$ function may be written as a function $K(x-y)$ of the difference of $x$ and $y$ where $x$ and $y$ traverse the real line (or the set of integers); $K(u)$ is then said to be a Polya frequency density of order $r\left(P F_{r}\right)$. In this case we assume $K(u)$ is integrable with respect to Lebesgue measure.

The theory of totally positive functions has been extensively applied in several domains of mathematics, statistics and mechanics. The notion of total positivity is a common thread running through many disciplines. They derive interest in their intrinsic relevance to the theory of stochastic processes of diffusion type [7]; in their natural occurrence in applications to statistics $[5 ; 6]$ and mechanics $[1]$; and in their elegant structural properties [8].

Examples of totally positive functions appear abundantly and naturally in different contexts. Most of the standard statistical distributions depending on a parameter such as the exponential family, 
noncentral $T, \mathfrak{X}^{2}$, correlation coefficient, etc. are $T P_{\infty}[\mathbf{5}]$.

Recently J. L. McGregor and this author [7] showed that the transition probability function $P(t, x, E)$ of a temporally homogeneous strong Markoff process $X(t)$ whose state space is the real line and which possesses a realization with continuous sample paths is totally positive in the variables $x$ and $E$. Specifically, if $x_{1}<x_{2}<$ $\cdots<x_{m}$ and $E_{1}<E_{2}<\cdots<E_{m}(E<F$ means that the set of points in $E$ all lie to the left of the set of points in $F$ ) then

$$
\operatorname{det} P\left(t, x_{i}, E_{j}\right) \geqq 0 \quad \text { for every } t>0 \text { and integer } m \text {. }
$$

Conversely under suitable mild smoothness conditions the inequalities (3) imply the existence of a realization of the process in which almost all path functions are continuous.

For birth and death stochastic processes the transition probability function $P_{i j}(t)$ is $T P_{\infty}$ in the variables $i$ and $j$ for every $t>0$. Other totally positive functions can be generated by the following composition law which expresses a continuous analogue for evaluating determinants arising through matrix multiplication. Let $X, Y$ and $Z$ represent linear sets. If $M(x, z)=\int K(x, y) L(y, z) d \sigma(y)$ converges absolutely where $\sigma$ is a sigma finite regular measure then

$$
\begin{gathered}
M\left(\begin{array}{c}
x_{1}, x_{2}, \cdots, x_{m} \\
z_{1}, z_{2}, \cdots, z_{m}
\end{array}\right)=\int_{y_{1}<y_{2}<\cdots<y_{m}}^{\ldots} \int K\left(\begin{array}{l}
x_{1}, x_{2}, \cdots, x_{m} \\
y_{1}, y_{2}, \cdots, y_{m}
\end{array}\right) \\
\cdot L\left(\begin{array}{l}
y_{1}, y_{2}, \cdots, y_{m} \\
z_{1}, z_{2}, \cdots, z_{m}
\end{array}\right) d \sigma\left(y_{1}\right) \cdots d \sigma\left(y_{m}\right), \\
x_{1}<x_{2}<\cdots<x_{m}, z_{1}<z_{2}<\cdots<z_{m}, x_{i} \in X, y_{i} \in Y, z_{i} \in Z .
\end{gathered}
$$

The following theorem provides a different kind of construction of $T P_{\infty}$ kernels. We state only the continuous form of this result.

TheOREM A. Let $X$ be a random variable whose density function $f(x)$ is a symmetric Pólya frequency function $P F_{\infty}$. Then the density function $f^{*}(x ; u)$ of the random variable $|X+u|(u>0$ is a parameter $)$ is $T P_{\infty}$.

We now describe several new classes of totally positive and sign regular functions that arise as absorption probabilities for stochastic processes whose one-step transition probability function is totally positive in the initial and final state variables. These totally positive functions relate to the time variable and one of the state variables of the process as contrasted with (3) which involves the initial and final state variables.

The following two statements are typical. 
THEOREM B. Let $\beta$ be a temporally homogeneous $T P_{r}$, Markoff chain (the transition probability matrix $P_{i j}$ is $T P_{r}, r \geqq 2$ ) whose state space is the non-negative integers. Then the n-step transition function $P_{0, j}^{n}$ is $T P_{r}$ in the variables $0 \leqq j<\infty$ and $n \geqq 0$.

Theorem C. Let 8 be a TP Markoff chain. Let $F_{i, j_{0}}^{n}$ denote the probability that first passage into the set of states $\leqq j_{0}$ occurs at the nth transition where the initial state of the process is $i>j_{0}$. Then $F_{i, j_{0}}^{n}$ is $T P_{r}$ in the variables $n \geqq 1$ and $i>j_{0}$.

These results are generalized in several different ways. By imposing further restrictions on the nature of the process such as spatial homogeneity, non-negative drift, etc. we can display additional sign regular functions constructed from various probability expressions natural to the process.

Further extensions to the case of discrete time continuous state $T P$, Markoff processes on the real line are available; continuous time analogs are also developed. As an example, we have

THEOREM D. Let $P_{i j}(t)$ be the transition density of $a$ birth and death process. Then the density function $q\left(t ; i, j_{0}\right)$ of the first passage time from state $i$ to state $j_{0}<i$ is $T P_{r}$ in the variables $t>0$ and $i>j_{0}$.

The theory can be extended to the case of linear diffusion processes. These processes are characterized by the Strong-Markoff property and continuity of sample paths.

We now indicate some implications of the preceding theorems for stochastic processes:

(a) Consider a $T P_{3}$ Markoff process on the non-negative real line. The $n$-step transition density $P^{n}(0, x)$ is unimodal as a function of $n$ for fixed $x>0$.

(b) The density function of the first passage time from 0 to the set $(x, \infty)$ is a unimodal function.

Similar conclusions are valid in the case of birth and death and linear diffusion processes. The result that the ergodic limits are approached monotonely emerges as a consequence of total positivity. We also obtain the following strong ratio theorem.

Theorem E. Let $P$ be a $T P_{2}$ temporally homogeneous Markoff chain whose state space is the non-negative integers with all states communicating. Suppose $\odot$ is recurrent; then for any four prescribed states $i, j, k, l$, $\lim _{n \rightarrow \infty} P_{k j}^{n} / P_{k l}^{n}$ exists, is finite and positive.

The proofs of these theorems, other results and applications will be published elsewhere. Theorem A was developed jointly by C. Stone and the writer. 


\section{BIBLIOGRAPHY}

1. F. Gantmacher and M. Krein, Oscillation matrices and kernels and small vibrations of mechanical systems, 2nd ed. (in Russian), Moscow-Leningrad, Gosudarstv. Izdat. Tehn.-Teor. Lit., 1950.

2. I. I. Hirschmann, Variation diminishing transformations and ultraspherical polynomials, J. Analyse Math., to appear.

3. - Variation diminishing Hankel transforms, J. Analyse Math., to appear.

4. I. I. Hirschmann and D. V. Widder, The convolution transform, Princeton, New Jersey, Princeton University Press, 1955.

5. S. Karlin, Decision theory for Pólya type distributions, case of two actions. I, Proceedings Third Berkeley Symposium on Probability and Statistics, Berkeley, California, University of California Press, Vol. I, 1956, pp. 115-129.

6. - Polya type distributions, II. Ann. Math. Statist. vol. 28 (1957) pp. 281-308.

7. S. Karlin and J. McGregor, Coincidence probabilities, Pacific J. Math. vol. 9 (1959) pp. 1141-1165.

8. I. J. Schoenberg, On Polya frequency functions. I, The totally positive functions and their Laplace transforms, J. Analyse Math. vol. I (1951) pp. 331-374.

STANFORD UNIVERSITY 